\title{
Experimental analysis of the influence of the impinged plate by an impinging jet on the vortex dynamics using PIV
}

\author{
Tarek Mrach ${ }^{1,}{ }^{*}$, Jana Hamdi $^{1}$, Kamel Abed-Meraïm ${ }^{1}$, Marwan Alkheir ${ }^{1}$, Hassan \\ Assoum $^{2}$, and Anas Sakout ${ }^{1}$ \\ ${ }^{1}$ Laboratoire des Sciences de l'Ingénieur pour l'environnement (LaSIE), University of La Rochelle, \\ 17000 La Rochelle, France \\ ${ }^{2}$ Beirut Arab University, Tripoli Branch, Liban
}

\begin{abstract}
In this experimental study, we are interested in the flow generated by a plane jet impacting on different plates. To analyse these flows and to compare the evolutions of the various parameters (velocities, turbulence rate, vortex structures, etc.), the PIV technique was used. For example, for a confinement ratio of 4 and a Reynolds number of 4838 , a comparison is made between the deviated flows for each of the three plates considered in this study. For the smooth slotted plate, it is noted that the large structures created by the shear between the jet and the driving zone are convected and are organized differently according to the initial dynamics of the flow (Re). Indeed, depending on the configuration, these large structures are either deviated laterally in the parietal flow or pass alternately through the slot. With regard to the solid plates, Kelvin Helmholtz's structures are always deflected, the parietal flow is more nourished but its vortex dynamics is influenced by the surface state. Indeed, for the smooth solid plate we have large swirl structures whereas for the corrugated plate, we notice the appearance of small vivid and intense vortex structures. A quantitative analysis of these phenomena is conducted in order to study and compare parietal flows.
\end{abstract}

\section{Introduction}

The impinging jets are widely used in many industrial and domestic applications. Thus, they are used both in cooling or drying in industry, for mixing and homogenization in the terminal units of conditioning systems and air ventilation in the transport or building. As a result, in addition to the analysis of their heat transfer capacities (flux, temperatures, ...). This study was undertaken to understand the influence of the nature of the plate struck by the jet on the vortex dynamics of the deviated parietal flow [1] [2].

\footnotetext{
*Tarek Mrach : tarek.mrach@gmail.com
} 


\section{Experimental apparatus}

The experimental setup consists of a part that generates the flow to be studied and a specific metrology part that allowed us to perform a 2D-2C PIV [3]. Thus, we were able to obtain the instant kinematic fields and compare the flows impacting the three different plates: a solid and smooth plate, a solid and corrugated plate and a slotted plate.

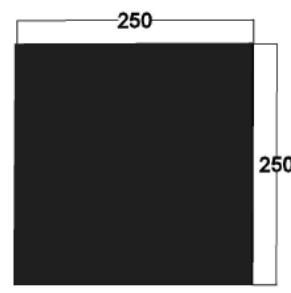

a)

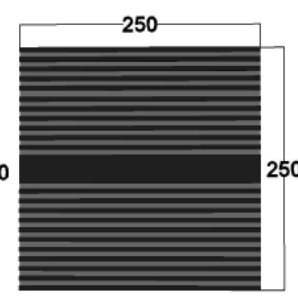

b)

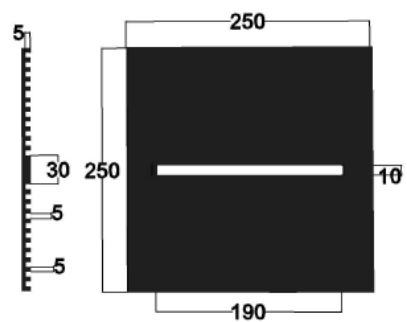

C)

Fig. 2. a) solid and smooth plate, b) solid and corrugated plate, c) slotted plate [mm]

\section{Results}

The analysis of kinematic fields allowed us to compare several parameters of these impacting flows. First, we were interested in transverse profiles of longitudinal velocities. Thus we were able to compare the lengths of the potential cores as well as the decrease of the velocities on the jet axis. Also, we studied the evolution of the turbulence rates during the progress of the jet and according to the increase of the Reynolds number. On the other hand, in order to quantify the vortex dynamics and particularly highlight the parietal flows, we have studied the evolution of large eddy structures (Kelvin Helmholtz structures). For example, we present in fig. 2 for each studied plate, on the left an instantaneous particular field and on the right an instantaneous kinematic field. Thus, we can notice that for the same Reynolds number we have vortex structures which are organized differently according to the impinged plate. In fact, for the smooth plate (a), they are larger and deviate in the transverse direction, for the corrugated plate (b), the structures are small, intense and close to the plate, whereas for the slotted plate (c) they hit the edge of the corrugations, are split and end up in the form of smaller structures [4].

To characterize the large vortex structures, to trace their trajectories and to carry out a temporal follow-up, we used the $\lambda_{2}$ criterion [5]. In figure 3 , we can see a representation of the positions of the vortex centres at different instants.

\section{Conclusion}

This comparative experimental study allowed the comparison between the flows generated by turbulent jets impinging different surfaces using a Laser metrology: the PIV. Thus we have shown, experimentally, that the vortex structures are small, intense and close to the wall for a corrugated plate, larger and deviated from the wall for a smooth plate and partly absorbed by the slot for a slotted plate. Also, the tangential velocities in the parietal flow are more intense in the case of the corrugated plate than for the smooth plate. This study continues with other objectives (influence of confinement, geometry of the ribs, ...). 


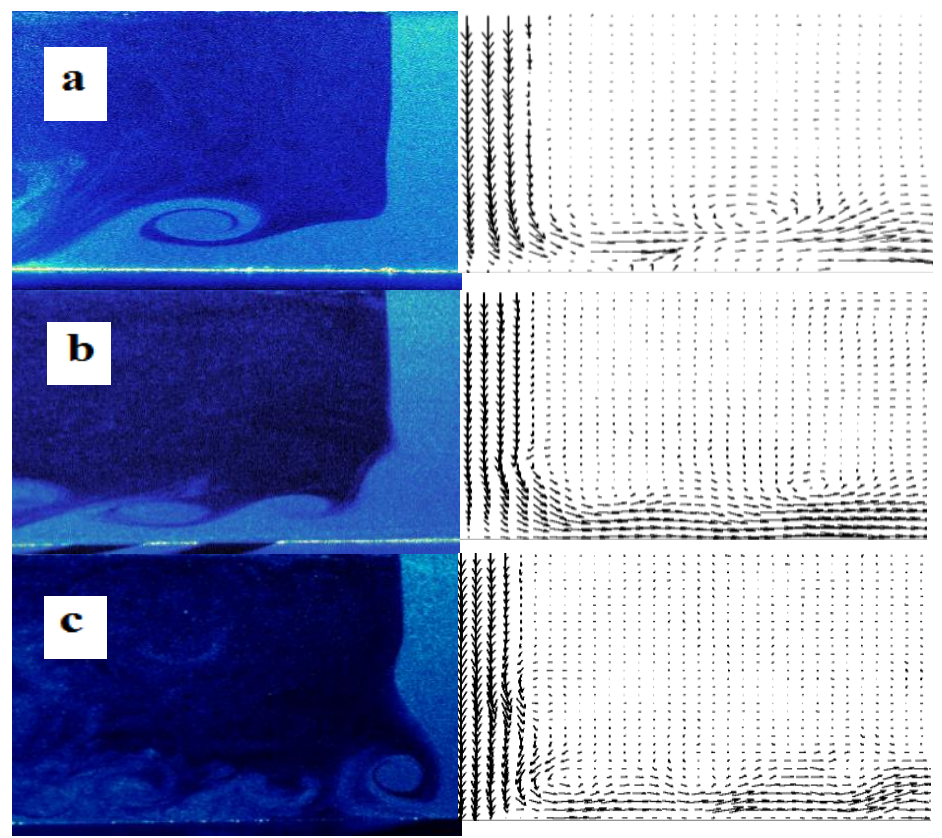

Fig. 2. Left: particular fields, right: their corresponding velocity vector fields. (a): solid and smooth plate, (b): solid and corrugated plate, (c): slotted plate.
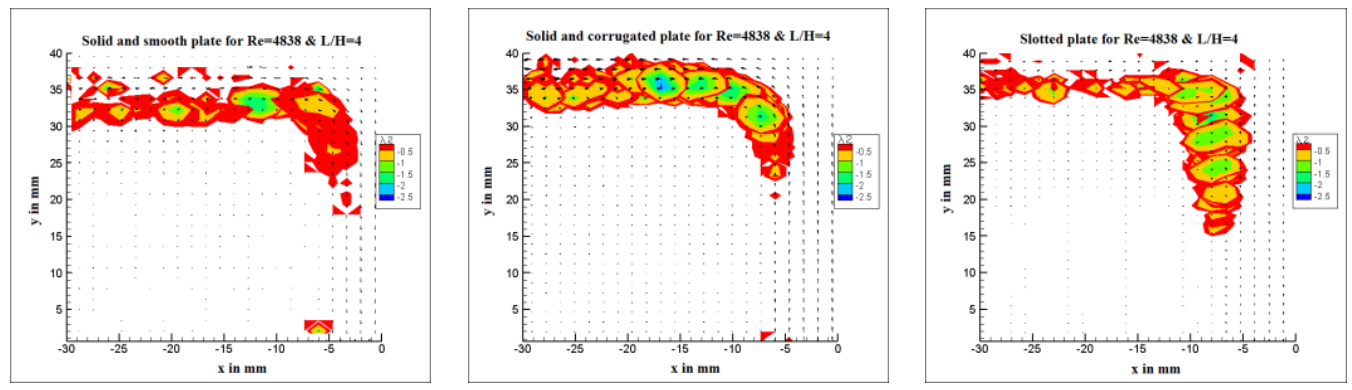

Fig. 3. Vortex trajectories for the three plates $(\mathrm{Re}=4838 \& \mathrm{~L} / \mathrm{H}=4)$

\section{Acknowledgments}

The authors wish to thank the region of Nouvelle Aquitaine for its financial support

\section{References}

1. J. Hamdi, PhD Thesis (Ecole Doctorale SI-MMEA, 2017)

2. H. Assoum, PhD Thesis (Ecole Doctorale SI-MMEA, 2013)

3. H.H. Assoum, M. El Hassan, K. Abed-Meraïm, R. Martinuzzi, A. Sakout, Fluid Dyn Res, 45, 045503 (2013)

4. H. H. Assoum, M. El Hassan, K. Abed-Meraim, et A. Sakout, Eur. J. Mech. - BFluids, 48, 231-235 (2014)

5. J. Jeong, F. Hussain, Fluid Mech, 285, 69-94 (1995) 